\title{
Über das Fixierungsmittel.
}

\section{Mitteilung. Diffusionsbeobachtung der Fixierungsmittel in Gelen nach Kohlrausch'scher Widerstandsbeobachtungsmethode.}

\author{
Von \\ cand. med. G. Yasuzumi. \\ Aus dem Anat. Institut der Mediz. Akademie zu Osaka. \\ (I.eiter: Prof. T. Tomita)
}

Mit 9 Textfiguren.

Es ist ausser Zweifel, dass die Fixierung in erster Reihe die totale Härtung, besonders Gerinnung der Zelle bedingt.

Jedoch, ob eine Härtung der Eiweisskörper der Zelle ohne Veränderung ihrer Form und ihrer Substanzmasse, sowie ihrer Struktur erreichbar ist, ist noch Sache der Diskussion.

Alfred Fischer nimmt die Stellung ein, dass manche Bilder in den fixierten und gefärbten Präparaten nicht dem ursprünglichen Zustande entsprechen, sondern ganz oder teilwiese Kunstprodukte sind.

R. Schäde hat in seinem Werke,,Vergleichende Untersuchung über Cytoplasma, Kern und Kernteilung in lebendem und in fixiertem Zustande" behauptet, dass Chromocentren Kunstprodukte sein können, ferner dass die Bilder der einzelnen Objekte nach Behandlung mit verschiedenen Fixierungsmitteln verschieden seien.

Moderne Botaniker und Anatomen wie G. Yamaha und T. Tomita nehmen einen grundsätzlich anderen. Standpunkt zur Fixierung des Gewebes ein. F. Cornu fand bei seiner Untersuchung bestätigt, dass die Härtung gleichzeitig von einer Schrumpfung des histologischen Präparates begleitet ist. Eine grosse Anzahl der Histologen ist aber der Ansicht, dass bei der Fixierung und Härtung im allgemeinen keine Veränderung 
in der Struktur des Präparates eintritt und wemn eine solche eintritt, diese lediglich in einer gleichmässigen Schrumpfung oder Volumverminderung infolge Wasserentziehung besteht, wodurch die Originalstruktur und das Bild des Präparates nicht gestört wird.

Auch Frank Schwarz hat gerüstähnliche Ausfälluugen durch Reagentien im Protoplasma und Zellsaft von Pflanzenzellen beschrieben und hält, es nicht für richtig, ,,aus den an fixierten Zellen auftretenden Bildern auf eine bestimmte Struktur zu schliesen."

Der Gedanke, dass das Protoplasma und seine lebenden Einschlüsse einen besonderen Bau besitzen, der demjenigen der kolloiden Körper ähnlich ist, wurde zum ersten Male von Nägeli ausgesprochen. Seit Nägeli wurde die Analogie zwischen den physikalischen Eigenschaften des Protoplasmas und denjenigen der Kolloide von Biologen mehrmals hervorgehoben. Als sich vereinzelte Stimmen erhoben, die darauf hinwiesen, dass die histologischen Strukturen des Protoplasmas nur eine Folge der Einwirkung der Reagentien auf die kolloiden Stoffe desselben darstellen können und dass eine einheitliche Protoplasmastruktur nicht existieren könne, wurde dies von der Mehrzahl der Naturforscher mit Misstrauen aufgenommen.

Erst im zwanzigsten Jahrhundert, nachdem die Kolloidchemie sich als ein selbständiger $Z_{w}$ weig der physikalischen Chemie entwickelt und die allgemeine Aufmerksamkeit auf sich gelenkt hatte, wurden die Anschauungen der Naturforscher notwendig zur Annahme einer Analogie zwischen dem physikalischen Zustand des Protoplasmas und dem kolloiden Zustand der Körper hingelenkt.

Kolloidchemisch betrachtet, können wir uns die Gewebe bestehend denken aus $\mathrm{I}$. irreversiblen, wenig elastischen Gelen,

II. reversiblen elastischen Gelen, III. Solen,

Übrings kann man keine scharfe Grenze zwischen reversiblen und irreversiblen Zustand im Protoplasma zeichen. Die Zwischenstufen aller Art werden vorhanden sein.

Die üblichen Zustandsveränderung kolloider Substanzen können in folgender Form ausdrückt werden ;

Festes Kolloid
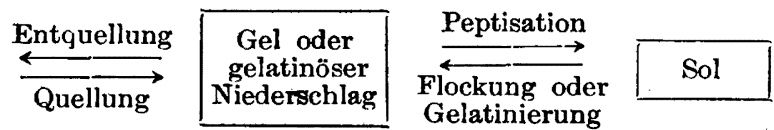

Die Fixierung bezweckt, die reversiblen Gelen und die Solen in den irreversiblen Gelenzustand zu überführen. 
Die Struktur von ,Solen“ ist wohl bekannt;

die ultramikrospische Untersuchung zeigt, dass das Kolloid aus zahlreichen Teilchen besteht, die sich in unabhängiger ,,Brownsche Bewegung" befinden.

In ,Gelen“ scheinen sich eben diese Teilchen zu verketten und dadurch eine Art festes Gitter zu bilden, denn das Gel besitzt Festigkeit und Elastizität ; doch scheinen dort zusammenhängende Flüssigkeitskanäle die Gele zu durchziehen, denn ein Gel hat eine hohe elektrische Leitfähigkeit. (Der Widerstand wurd in der üblichen Weise durch eine Wheatstonesche Brückenanordung gemessen, die Widerstandskapazität des Elektrodengefässes betrug C:0,1811 20․ C.)

Die ersten Versuche sollen die elektrischen Leitvermögen der 2\%igen Agarlösung durch diese Methode zeigen.

Tabelle I gibt die Ergebnisse.

Tabelle 1

\begin{tabular}{c|c|c|c}
\hline $\begin{array}{c}\text { Temperatur in } \\
\text { Graden Celsius }\end{array}$ & Kolloid Zustand & Zeit & Leitvermögen \\
\hline 58.0 & Sol & 0. & 0.008387 \\
32.0 & Gel & 1. st. & 0.006614 \\
32.0 & Gel & 2. " & 0.006614 \\
32.0 & Gel & $5 .$, & 0.006614
\end{tabular}

Es, ist bekannt, dass das Leitvermögen aller Elektrolyte beträchtlich mit steigender Temperatur wächst. Diese Beobachtung bezeigt die Verschiedenheit des Leitvermögens nach Kolloidzustand.

So erkennen wir, dass bei der Fixierung das Gewebe sich ausser dem Kolloidzustand noch in seinen physikalichen Eigenschaften verändert. Weiter wollte ich eine kleine Untersuchung über Diffusionserscheinung der Fixierungsmittel mitteilen. Die Kenntnis des Fixierungsmittels, besonders der Diffusionsvorgänge ist für die Histologen bei der Zellforschung von besonderen Wichtigkeit. „Ohne Diffusion gibt es keine Fixation.“ Die Untersuchungen der Diffusionsvermögen der verschiedenen Fixierungsmittel wurden von folgenden Forschern Tomita, Fujita, Wasielewski, Schaffer, Tellyesniczky, Gerota, Beijerinck und Yasuzumi gemacht.

Tellyesniczky hat eine systematische Untersuchung über die Diffusion der bekanntesten Fixierungsflüssigkeiten an verschiedenen 
parenchymatösen Organen gemacht. Die erste Diffusionsuntersuchung von elektrischen Lösungen nach Kohlrausch'scher Methode wurde von Tomita und Fujita gemacht, welche zeigt, dass die Elektrolyten nicht nur in die Gewebe diffundieren, sondern auch nach kurzer Zeit aus den Geweben ausscheiden. In meiner früheren Arbeit wurden eine Reihe von Beobachtungen über die physikalischen chemischen Wirkung der verschiedenen Fixierungsmittel auf das Gewebe, besonders unter Berücksichtigung ihrer verschiedenen Eigenschaften, wie des osmotischen Druckes, der Fällungskraft sowie der Diffusionsgeschwindigkeit gezeigt.

Mancher Forscher hat über die Ursache der Quellung und Schrumpfung der Gewebe durch die verschiedenen Fixierungsmittel Mitteilungen gemacht. (Sjöbering, Mann, Vermaat, Dekhuyzen, Stoeltzner, Tobler, Höber, Bito, Schaffer, Bottazzi, W. Berg, F. Blum, Hamburger, Sorger, Studnicka, u.a.) Auch sollen bei der Fixierung die Gewebe in ihren chemischen Eigenschaften nicht zu sehr verändert sein. Eine grosse Anzahl der Histologen berücksichtigt nicht, dass bei der Fixierung die Fixierungsmittel allmählich stets getrübt werden, und dass die Farbe derselben sich verändert. Die Trübung und Farben-veränderung der Fixierungsmittel bezeigt die grosse chemische Veränderung der Objekte d.h. Zerstörung des Gewebes.

Daher ist es von besoderer Bedeutung und von Interesse, die Diffusionserscheinungen der Fixierungsmittel mit Rücksicht auf die Zeit zu beobachten. Man wird die Diffusionsversuche in der Gallerte im allgemeinen in folgender Weise aufstellen: man füllt Proberöhrchen mit einer möglichst wässrigen (20\%igen Gelatine oder 2\%igen Agar) Gallerte, giesst nach dem Erstarren die zu untersuchende Lösung darauf. Nach bestimmter Zeit wird etwas in die Gallerte diffundiert sein. In vielen Fällen wird man durch dem Augenschein (z.B. bei Fafbstoffen), durch Indikator oder durch Fällungsreaktion erkennen, ob und wie etwas von der Aussenflüssigkeit eindiffundiert ist.

Es käme noch in Betracht, dass man nicht die Menge der in die Gallerte eindiffundierten Substanz bzw. deren Diffusionsweg bestimmt, sondern den Verlust der überstehenden Flüssigkeit an Substanz.

Wenn das Volumen der noch Prozent und elektrischen Widerstand bekannten Lösungen in die Gallerte diffundiert, so nimmt die Konzentration der überstehenden Flüssigkeit $a b$, umgekehrt aber steigert sich der elekt. Widerstand.

Zur Widerstandsbestimmung mit Wechselstrom dient stets eine Arrhenius'sches Widerstandsgefäss, meistens mittels der Wheatstoneschen Brücke. 
Tebelle 1.

Widerstand von $10 \mathrm{ccm}$ Wasser der Wasserleitung auf 20\%ige Gelatine $\left(20^{\circ} . \mathrm{C}\right)$.

\begin{tabular}{l|r|r|r}
\hline $\mathbf{T}$ & $\mathbf{R}$ & $\log \mathbf{T}$ & $\log \mathbf{R}$ \\
\hline 0, & 1960,38 & & \\
0,5 & 350,20 & $-0,30103$ & 2,5443161 \\
1,0 & 254,64 & 0,00000 & 2,4059266 \\
2,0 & 221,86 & 0,30103 & 2,3450790 \\
4,0 & 180,60 & 0,60206 & 2,2567177 \\
8,0 & 119,04 & 0,90309 & 2,0756929 \\
10,0 & 92,88 & 1,00000 & 1,9679222 \\
20,0 & 88,21 & 1,30103 & 1,9455178
\end{tabular}

Tabelle 2.

Widerstand von $10 \mathrm{ccm}$ dest. Wasser auf 20\% ige Gelatine $\left(20^{\circ} . \mathrm{C}\right)$

\begin{tabular}{c|r|r|c}
\hline \multicolumn{1}{l|}{$\mathrm{T}$} & $\mathrm{L}$ & $\mathrm{Log} \mathrm{T}$ & \\
\hline 0, & $>11100,00$ & & \\
0,5 & 391,60 & $-3,30103$ & 2,5928427 \\
1,0 & 336,60 & 0,00000 & 2,5271141 \\
2,0 & 230,92 & 0,30103 & 2,3634615 \\
4,0 & 179,22 & 0,60206 & 2,2533865 \\
8,0 & 128,92 & 0,90309 & 2,1106570 \\
10,0 & 116,28 & 1,00000 & 2.0655050 \\
20,0 & 78,88 & 1,30103 & 1,8970770
\end{tabular}


Tabelle 3.

Widerstand von $10 \mathrm{ccm}$ konzentrierter Sublimatlösung auf 20\% ige Gelatine $\left(18^{\circ} . \mathrm{C}\right)$

\begin{tabular}{c|c|c|c}
\hline $\mathrm{T}$ & $\mathrm{R}$ & $\mathrm{Log} \mathrm{T}$ & Log R \\
\hline 0, & 1044,99 & & \\
0,5 & 246,10 & $-0,30103$ & 2,3911116 \\
1,0 & 171,10 & 0,00000 & 2,2332500 \\
2,0 & 128,96 & 0,30103 & 2,1104550 \\
4,0 & 95,10 & 0,60206 & 1,9781805 \\
8,0 & 73,58 & 0,90309 & 1,8667598 \\
10,0 & 69,08 & 1,00000 & 1,8393528 \\
20,0 & 55,56 & 1,30103 & 1,7447622
\end{tabular}

Tabelle 4.

Widerstand von $10 \mathrm{ccm} \mathrm{n} / 10$ Sublimatlösung auf $20 \%$ ige Gelatine $\left(20^{\circ} . \mathrm{C}\right)$

\begin{tabular}{c|c|c|c}
\hline $\mathrm{T}$ & $\mathbf{R}$ & $\operatorname{Iog} \mathrm{T}$ & $\operatorname{Iog} \mathrm{R}$ \\
\hline 0, & 1232,00 & & \\
5,0 & 257,04 & $-0,30103$ & 2,4090007 \\
1,0 & 214,36 & 0,00000 & 2,3311437 \\
2,0 & 158,00 & 0,30103 & 2,7986571 \\
4,0 & 117,60 & 0,60206 & 2,0482864 \\
8,0 & 96,08 & 0,90309 & 1,9826330 \\
10,0 & 83,46 & 1,00000 & 1,9215824 \\
20,0 & 72,52 & 1,30103 & 1,8604578
\end{tabular}


Tabelle 5 .

Widerstand von $10 \mathrm{ccm} \mathrm{n} / 10$ Sublimatlösung auf 2\%iges Agar $\left(20^{\circ} . \mathrm{C}\right)$

\begin{tabular}{c|c|c|c}
\hline $\mathrm{T}$ & $\mathrm{R}$ & $\log \mathrm{T}$ & $\log \mathrm{R}$ \\
\hline 0, & 1981,51 & & \\
0,5 & 1680,62 & $-0,30103$ & 3,2254644 \\
1,0 & 1472,94 & 0,00000 & 3,1671733 \\
2,0 & 1318,20 & 0,30103 & 3,1199813 \\
8,0 & 1085,00 & 0,90309 & 3,0354297 \\
13,0 & 966,57 & 1,11394 & 2,9852333 \\
21,89 & 828,32 & 1,32366 & 2,9181981
\end{tabular}

Morse und Piere haben folgende Gleichung für die Bildung der Bänder (Ringe) des Silberchromates in Röhrchen aufgestellt: (Liesegang'scher Ring)

$$
\frac{\mathrm{h}}{\sqrt{\mathrm{t}}}=\mathrm{K}
$$

wo $t$ die Entstehungszeit eines Ringes und $\mathrm{h}$ seine Höhe über der Basis des Röhrchens bedeuten. Diese Gleichung wurde von anderen Autoren auf das vollkommenste bestätigt gefunden; kurz nachher hat Hausmann durch Traube angeregt, dieselbe Gleichung zum Berechnen der Diffusionsgeschwindigkeit von Silbernitrat in einer mit Kaliumchlorid versetzen Gelatinegallerte angewandet und sie völlig richtig befunden. In der Untersuchung von Tomita und Fujita wurde die grosse Abhängigkeit des elektrischen Widerstandes von der Konzentration der Lösung gezeigt. Auch M. Watabe hat folgende Gleichung für die Diffusionsgeschwindigkeit von Silbernitrat in einer mit Chlor vermischten Gelatinegallerte in Röhrchen aufgestellt;

$$
\frac{d}{\sqrt{t}}=a+b t
$$

$\mathrm{d}=$ Diffusionsabstand

$t=$ Diffusionszeit

a und $b$ Konstante 
Die Diffusionserscheinung der Elektrolyten in überschichtete dest. Wasser, Wasser der Wasserleitung, n/10 Sublimatlösung und konzentrierte Sublimatlösung von 20\% Gelatine oder 2\% Agar-Gallerte erfolgt streng gesetzmässig. Die graphische Darstellung drückt die bestimmte Kurve aus.

Es ist daher von besonderem Interesse, diese Gesetzmässigkeit auch zahlenmässig $\mathrm{zu}$ formulieren.

Wenn man mit $\mathrm{R}$ den elekt. Widerstand der Lösung, mit $\mathrm{T}$ die Diffusionszeit und mit $\mathrm{C}$ den elekt. Widerstand vor der Diffusion bezeichnet, so wird demnach

$$
\mathrm{C}-\mathrm{R}=\mathrm{a} \mathrm{T}^{\mathrm{b}}
$$

für die rechnerische Darstellung der Diffusionsvorgậnge wird obige Gleichung logarithmiert;

$$
\text { man erhält so; } \log \mathrm{C}-\log \mathrm{R}=\log \mathrm{a}+\mathrm{b} \log \mathrm{T}
$$

Diese ist die Gleichung einer Geraden. a und $b$ Konstante

Ist. die zu untersuchende Flüssigkeit das dest. Wasser und nehmen wir für C den Wert $\infty$, so folgt

$$
\mathrm{R}=\mathrm{a} \mathrm{T}^{-\mathrm{b}}
$$

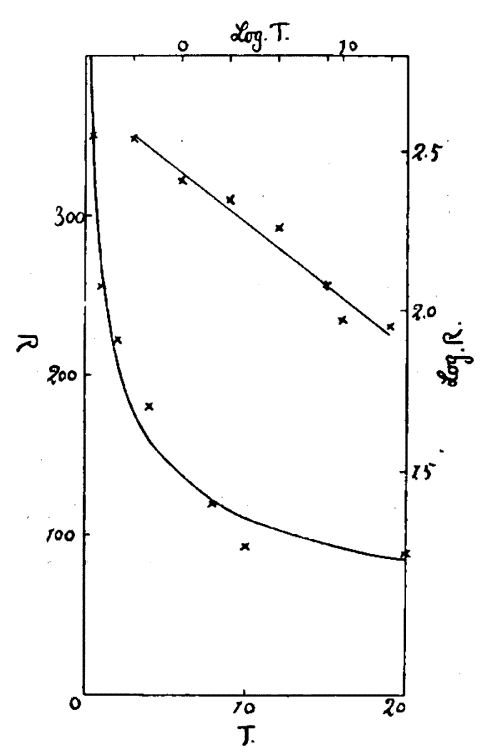

Fig. 1.

Widerstand von 10 ccum Wasser der Wasserleitung auf $20 \%$ Gelatine.

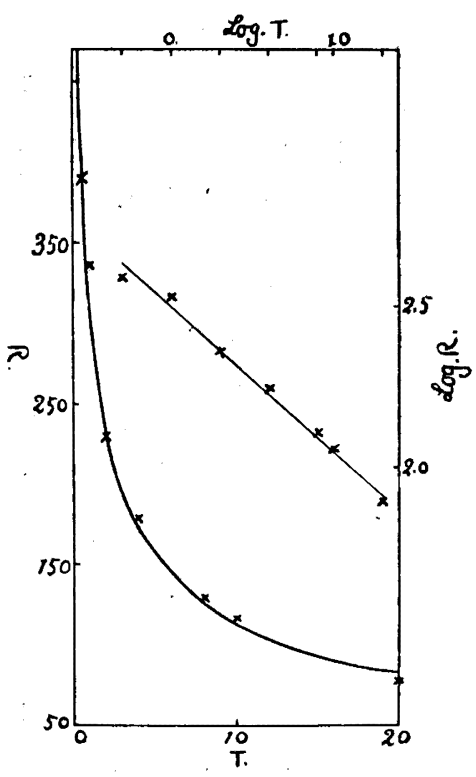

Fig. 2

Widerstand von $10 \mathrm{ccm}$ dest. Wasser auf $20 \%$ Gelatine. 


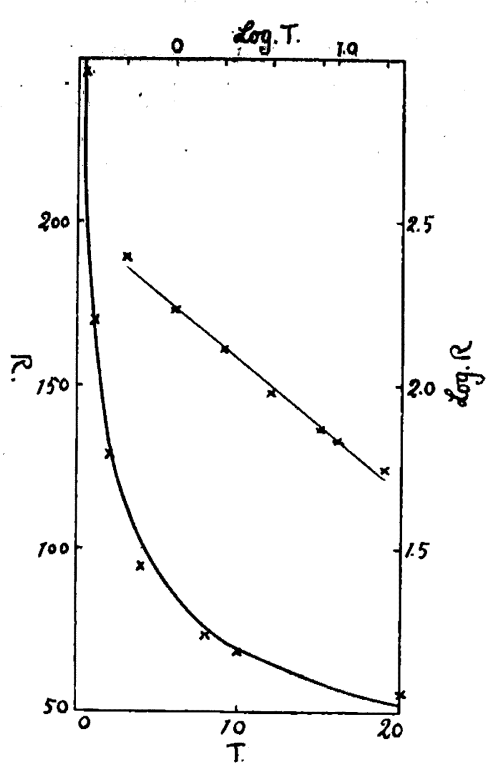

Fig. 3.

Widerstand von $10 \mathrm{ccm}$ konz. Sublimatlösung auf $20 \%$ Gelatine.

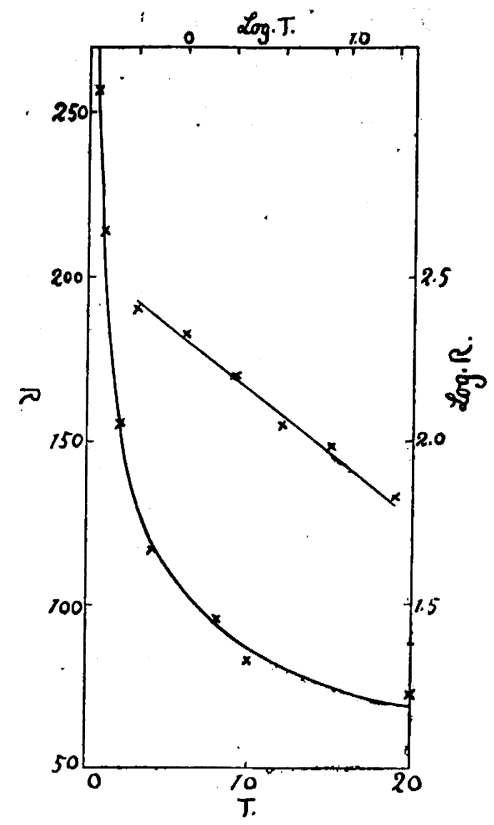

Fig. 4.

Widerstand von $10 \mathrm{ccm}$ Sublimatlösung auf 20\% Gelatine.

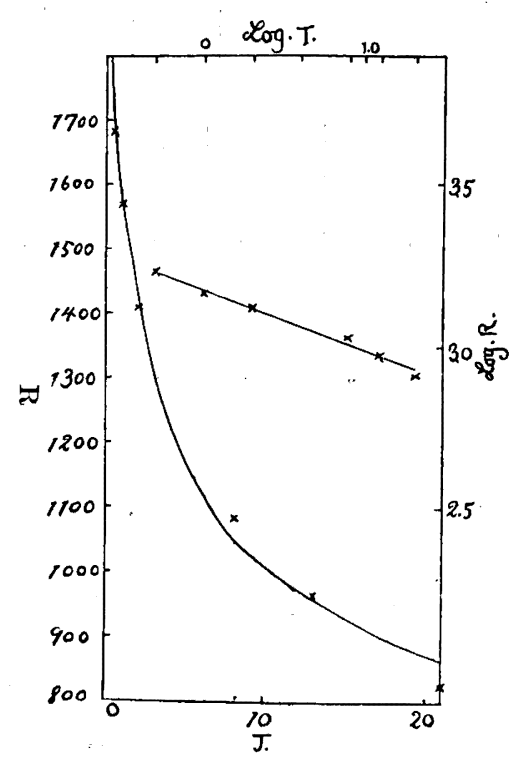

Fig. 5.

Widerstand von $10 \mathrm{ccm} \mathrm{n} / 10$ Sublimatlösung auf $2 \%$ Agar. 
Die zwei senkrechten Achsen in den Figuren zeigen den elektrischen Widerstand(R) und Log. R. Die zwei Querachsen zeigen die Diffusionszeit(T) und Log. T. Aus der folgenden Formel (Methode der kleinsten Quadrate und Ausgleichungsmethode) findet man den Wert der Konstante $a$ und $b$.

$$
\begin{aligned}
& a=\frac{\sum_{k=1}^{n} X_{k} \sum_{k=1}^{n} X_{k} y_{k}-\sum_{k=1}^{n} y_{k} \sum_{k=1}^{n} X_{k}^{2}}{\left(\sum_{k=1}^{n} X_{k}\right)^{2}-n \sum_{k=1}^{n} X_{k}^{2}} \\
& b=\frac{\sum_{k=1}^{n} X_{k} \sum_{k=1}^{n} y_{k}-n \sum_{k=1}^{n} X_{k} y_{k}}{\left(\sum_{k=1}^{n} X_{k}\right)^{2}-n \sum_{k=1}^{n} X_{k}^{2}}
\end{aligned}
$$

Ausgleichungsmethode :

$$
a t+b \gamma+c=0
$$

z.B.

\begin{tabular}{|c||c|c|c|c|c|c|}
\hline$t$ & $t_{1}$ & $t_{2}$ & $t_{3}$ & $t_{4}$ & $t_{5}$ & $t_{6}$ \\
\hline$\gamma$ & $\gamma_{1}$ & $\gamma_{2}$ & $\gamma_{3}$ & $\gamma_{4}$ & $\gamma_{5}$ & $\gamma_{6}$ \\
\hline
\end{tabular}

\begin{tabular}{|c||c|c|c|c|}
\hline$t$ & $t_{1}$ & $t_{2}$ & $t_{3}$ & $\frac{1}{3} \Sigma t^{\prime}$ \\
\hline$\gamma$ & $\gamma_{1}$ & $\gamma_{2}$ & $\gamma_{3}$ & $\frac{1}{3} \Sigma \gamma^{\prime}$ \\
\hline
\end{tabular}

\begin{tabular}{|c|c|c|c|c|}
\hline$t$ & $t_{4}$ & $t_{5}$ & $t_{6}$ & $\frac{1}{3} \Sigma t^{\prime \prime}$ \\
\hline$\gamma$ & $\gamma_{4}$ & $\gamma_{5}$ & $\gamma_{6}$ & $\frac{1}{S} \Sigma \gamma^{\prime \prime}$ \\
\hline
\end{tabular}

$$
\begin{aligned}
& \frac{\mathrm{a}}{3} \sum \mathrm{t}^{\prime}+\frac{\mathrm{b}}{3} \sum \gamma^{\prime}+\mathrm{c}=0 \\
& \frac{\mathrm{a}}{3} \sum \mathrm{t}^{\prime \prime}+\frac{\mathrm{b}}{3} \sum \gamma^{\prime \prime}+\mathrm{c}=0
\end{aligned}
$$

Aus der obigen Gleichung (1) und (2) findet man den Wert der Konstante a und b. $\quad c=$ der elekt. Widerstand vor der Diffusion.

1. dest. Wasser (20\% Gelatine) $\ldots . . .1060,38-\mathrm{R}=7,2482 \mathrm{~T}^{0.3898}$

2. Wasser $d$. Wasserleitung (20\% Gelatine)...R $=292,63 \mathrm{~T}^{-0.4400}$

3. konz. Sublimatlösung (20\% Gelatine) $\ldots 1044,99-\mathrm{R}=5,9911 \mathrm{~T}^{0.4038}$

4. $\mathrm{n} / 10$ Sublimatlös. (20\% Geqlatine) $\ldots 1230,00-\mathrm{R}=5,9837 \mathrm{~T}^{0.3938}$

5. n/10 Sublimatlös. (20\% Gelatine) $\ldots 1981,51-\mathrm{R}=1,6900 \mathrm{~T}^{0.14475}$ 
Ich stelle $\operatorname{nur}\left(\frac{\Delta}{\mathrm{R}}\right)^{2}$ von $1044,99-\mathrm{R}=5,9911 \mathrm{~T}^{0.4038}$ dar, lasse $\left(\frac{\Delta}{\mathrm{R}}\right)^{2}$ der anderen Gleichungen unberücksichtigt.

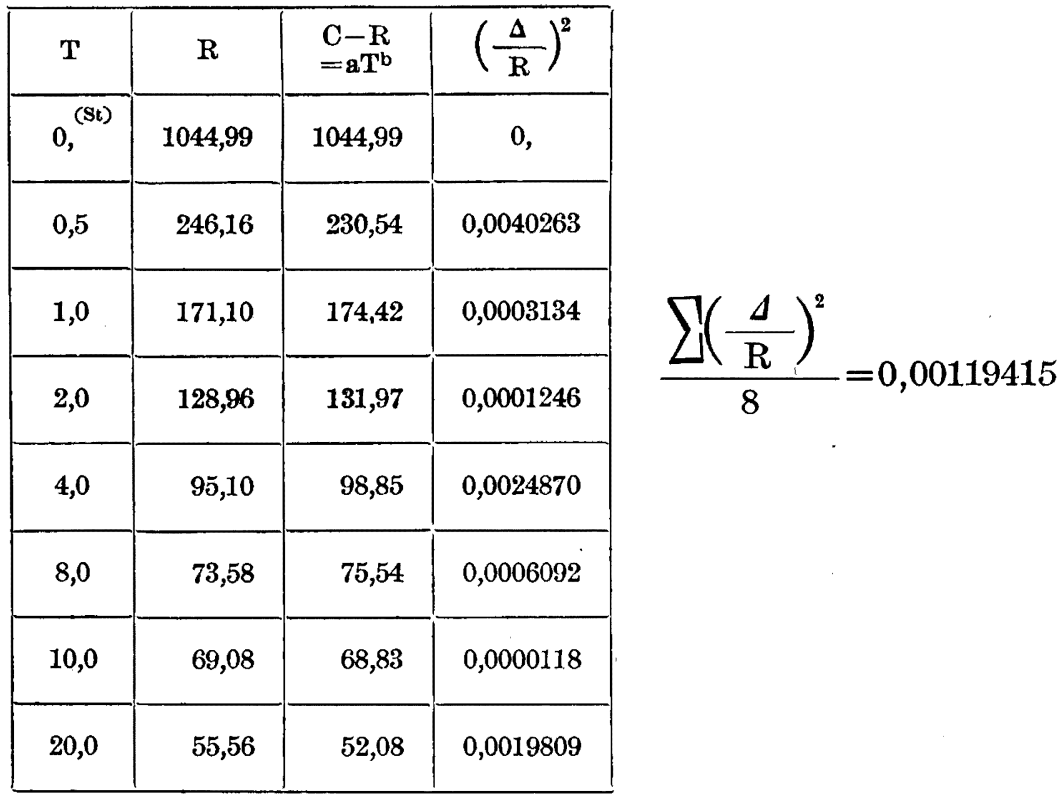

Tabelle 6.

Widerstand von konz. Pikrinsäurelösung auf 20\%ige Gelatine $\left(20^{\circ} .0 \mathrm{C}\right)$

\begin{tabular}{|c|c|c|c|c|c|c|c|c|c|c|}
\hline Zeit & 0 & $5^{\mathrm{m}}$ & $10^{\mathrm{m}}$ & $20^{\mathrm{m}}$ & $30^{\mathrm{m}}$ & $1^{\mathrm{st}}$ & $2^{\mathrm{St}}$ & $4^{\mathrm{st}}$ & $8^{\mathrm{st}}$ & $10^{\mathrm{St}}$ \\
\hline W.S & 11,568 & 12,242 & 12,268 & 13,130 & 13,716 & 16,160 & 19,190 & 25,080 & 42,590 & 44,900 \\
\hline
\end{tabular}

Bei der Diffusionserscheinung von konz. Pikrinsäurelösung in 20\%iger. Gelatine findet man folgende Gleichung aus der graphischen Darstellung.

$$
\mathrm{R}=\mathrm{a}+\mathrm{bT} \text {. }
$$




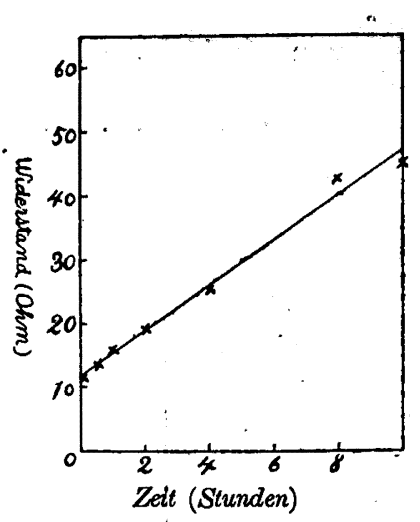

Fig. 6.

IViderstand von $10 \mathrm{ccm}$ konz. Pikrinsäurelösung auf $20 \%$ Gelatine.

Durch die Methode der kleisten Quadrate findet man den Wert der Konstante a und $b$.

$$
\text { also } R=12,104+3,470 \mathrm{~T}
$$

\begin{tabular}{|c|c|c|c|c|c|c|c|c|c|c|}
\hline $\mathrm{W} . \mathrm{S}$ & 11,568 & 12,242 & 12,268 & 13,130 & 13,716 & 16,160 & 19,190 & 25,080 & 42,590 & 44,900 \\
\hline $\begin{array}{c}\mathrm{R}= \\
\mathrm{a}+\mathrm{bT}\end{array}$ & 12,104 & 12,394 & 12,684 & 13,264 & 13,839 & 15,574 & 19,044 & 25,984 & 39,864 & 42,574 \\
\hline$\Delta$ & $-0,536$ & $-0,152$ & $-0,416$ & $-0,134$ & $-0,123$ & $-0,586$ & $+0,046$ & $-0,904$ & $+2,726$ & $+2,326$ \\
\hline
\end{tabular}

Man füllt die Schale (Radius; $6.0 \mathrm{~cm}$ ) mit einer wässrigen (20\%igen Gelatine oder 2\%igen Agar) Gallerte, giesst nach dem Erstarren die verschiedenen Konzentrationslösungen von Kalium Bichromat darauf. Fig 7. und 8. (2\% Agar), Fig 9. (20\% Gelatine), Tabelle 7. (2\% Agar). und Tabelle 8 (20\% Gelatine) geben die Ergebnisse.

Tabelle 7

\begin{tabular}{|c|c|c|c|c|}
\hline \multirow{2}{*}{ Zeit } & \multicolumn{4}{|c|}{ Elektrischer Widerstand (Ohm) } \\
\cline { 2 - 5 } & $3,44 \%$ & $3,0 \%$ & $1.5 \%$ & $0.6 \%$ \\
\hline 0, & 8,162 & 8,649 & 15,30 & 35,64 \\
$\frac{1}{3}$, & - & - & 20,00 & - \\
$\frac{1}{2}$, & 10,202 & - & - & 47,37 \\
$\frac{2}{3}$, & - & 10,760 & 22,82 & - \\
1,0 & 11,412 & 13,160 & 22,82 & 51,35 \\
\hline
\end{tabular}


Utber das Fixierungsmittel.

\begin{tabular}{|c|c|c|c|c|}
\hline \multirow{2}{*}{ Zeit } & \multicolumn{3}{|c|}{ Elektrischer Widerstand (Ohm) } \\
\cline { 2 - 5 } & $3,44 \%$ & $3,0 \%$ & $1.5 \%$ & $0,6 \%$ \\
\hline 2,0 & 11,880 & 13,832 & 23,92 & 53,00 \\
4,0 & 11,818 & 13,664 & 24,38 & 53,11 \\
6,0 & - & - & 26,00 & 55,44 \\
8,0 & 12,480 & 13,609 & 24,47 & $(61,61$ Zeit $=7$ st $)$ \\
10,0 & 12,720 & - & 24,34 & 55,44 \\
\hline Temp. & $22^{\circ} . \mathrm{C}^{\prime}$ & $22^{\circ} . \mathrm{C}$ & $22^{\circ} \cdot \mathrm{C}^{\prime}$ & 54,45 \\
\hline
\end{tabular}

Tabelle 8. (22․ C)

\begin{tabular}{|c|c|c|c|c|c|c|c|}
\hline Zeit & $0^{\mathrm{St}}$ & 0,5 & $1,0^{\mathrm{St}}$ & $2,0^{\mathrm{St}}$ & 4,0 & $8,0^{\mathrm{St}}$ & 10,0 \\
\hline W.S & 8,160 & 9,000 & 9,459 & 10,161 & 10,736 & 10,483 & 10,417 \\
\hline
\end{tabular}

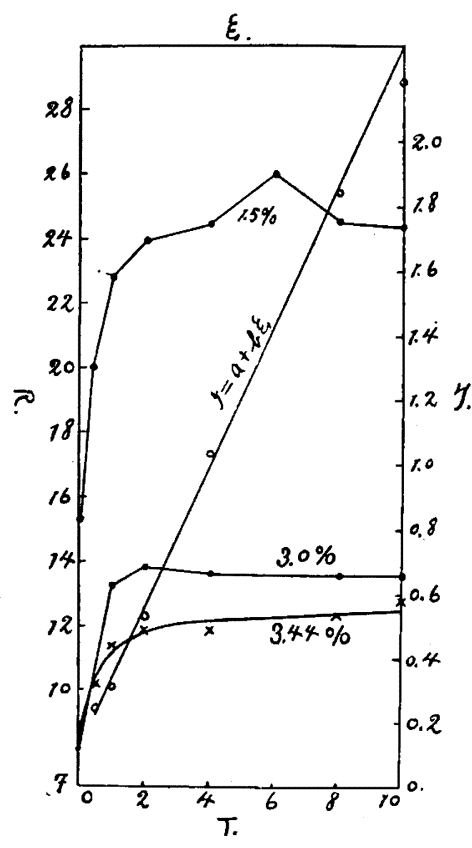

Fig. 7.

Widerstand von verschied. Konzentrationslōsungen von Kalium Bichromat $(10 \mathrm{ccm})$ auf $2 \%$ Agar.

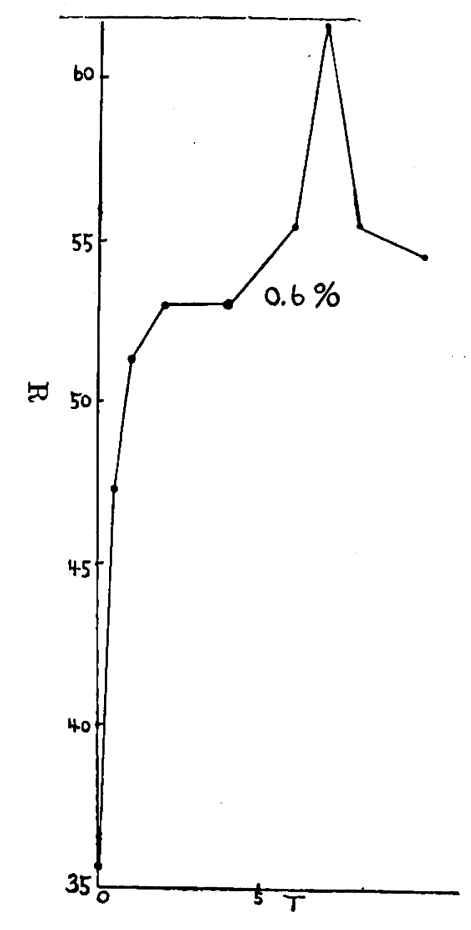

Fig. 8.

Widerstand von $10 \mathrm{ccm} 0,6 \%$ K. Bichromatlösung auf $2 \%$ Agar. 


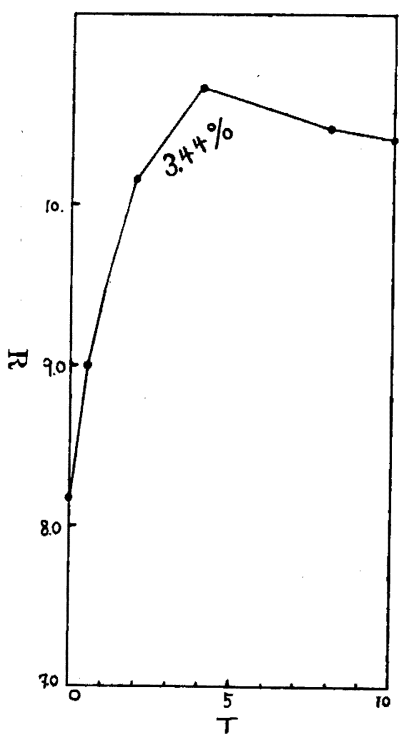

Fig. 9.

Der steile Anstieg des Widerstandes ist charakteristisch für eine Konzentrations veränderung der Kaliumbichromatlösung.

Bei 3\%, 1,5\% und 0,6\% Kaliumbichromatlösung nimmt der elektrische Widerstand nach bestimmter Zeit ab. Das bedeutet, dass die Elektrolyten (wie Eiweisskörper und Salz) von der Gellatinegellerte in Kaliumbichromatlösung diffundieren. Daher spielt die Konzentration des Fixierungsmittels eine wichtige Rolle bei der Fixierung des Gewebes.

Die untereste Kurve in Fig. 7. zeigt, wie sich der Widerstand in 3,44\% Kaliumbichromatlösung mit dem Zeitverlauf ändert.

Wenn man mit $\mathbf{T}$ die Diffusionszeit, mit $\mathrm{R}$ den elektrischen Widerstand bezeichnet, so wird demnach :

$$
\begin{aligned}
& \left.\begin{array}{l}
\mathrm{R}=\mathrm{c}+\frac{\mathrm{T}}{\mathrm{a}+\mathrm{bT}} \mathrm{a}, \mathrm{b} \text { und } \mathrm{c} \text { konstante } \\
\mathrm{T}=0 \\
\mathrm{R}=8,162
\end{array}\right\} \text { also } \mathrm{c}=8,162
\end{aligned}
$$

Wenn man $y=\frac{T}{R-8,162} \quad \xi=T$ setzt, so wird demnach, $y=a+b \xi$

\begin{tabular}{|c|c|c|c|c|c|c|c|}
\hline$\xi$ & 0 & $0,5^{\mathrm{St}}$ & $1,0^{\mathrm{st}}$ & $2,0^{\mathrm{st}}$ & $4,0^{\mathrm{st}}$ & $8,0^{\mathrm{st}}$ & $10,0^{\mathrm{St}}$ \\
\hline $\mathrm{y}$ & 0 & 0,2463 & 0,3077 & 0,5376 & 1,041 & 1,8527 & 2,1939 \\
\hline
\end{tabular}

aus der empirischen Formel findet man

$$
\begin{aligned}
& \mathrm{y}=0,1164+0,2188 \xi \\
& \text { also } \mathrm{R}=8,162+\frac{\mathrm{T}}{0,1164+0,2188 \mathrm{~T}}
\end{aligned}
$$

Die Diffusion von 3,44\% igen Kaliumbichromatlösung erfolgt regelmässig, d.h. mit der Zeit nimmt sie erst schnell, dann immer langsamer $\mathrm{zu}$.

Es ist bekannt, dass beim Fixierungsprozesse im übrigen allerei 
Strukturen stets mehr oder minder einen Substanzverlust infolge erhöhter Permeabilität erfahren, insbesendere zeigt sich das bei der Anwendung von Fixierungsmitteln, die reich an Säure oder lipoidlöslichen Stoffen (z.B. Alkohol, Äther, Chloroform) sind. Ich glaube, meine kleine Untersuchung hat den sicheren Beweis für den Substanzverlust bei der Fixierung mit Sublimatlösung ergeben. Die Fixierung mit Sublimatlösung aber wird von einer grossen Anzahl von Histologen empfohlen.

G. Yamaha hat in seinem Werke, ,Über die Lebendbeobachtung der Zellstrukturen, nebst dem Artefaktproblem in Pflanzytologie“ behauptet, dass die Anastomose des Karyotius als eine Folge des von Substanzverlust (auch. Entwässerung) begleiteten ,Phasenumschlags“ aufgefasst werden kann.

So gibt es kein Fixierungsmittel, dass ein in jeder Hinsicht befriedigendes Ergebnis zu liefern umstande wäre.

Fixierung mit Fixierungsmitteln ist die Gerinnung der Eiweisskörper der Zelle, die auch von Zerstörung des Gewebes begleitet ist.

Die Struktur, die nach Fixierung, Härtung und Färbung erreicht wird, ist der lebenden Struktur ganz ungleich.

Kurze Zusammenfassung der wichtigsten Ergebnisse :

1. Bei der bestimmten Konzentration erfolgt die Diffusion des Fixierungsmittels in Gelen und auch die Ausscheidung der Eiweisskörper, Salze u.a. aus Gelen streng regelmässig.

2. Der Substanzverlust bei der Fixierung mit Sublimatlösung wird durch die folgende Gleichung dargestellt.

$$
\mathrm{c}-\mathrm{R}=\mathrm{aT}
$$

$\mathrm{c}=$ der elektrische Widerstand vor der Diffusion

$\mathrm{R}=$ elektrischer Widerstand

$\mathrm{T}=$ Zeit

a und b. Konstante

Daher erfolgt der Substanzverlust bei der Fixierung mit Sublimatlösung streng regelmässig, d.h. mit der Zeit nimmt er erst schnell, dann immer langsamer zu.

3. Die Ausscheidungskurve, welche die Elektrolyten aus den Gelen in überstehendem dest. Wasser ausscheiden, verlangt eine andere mathematische Formulierung, welche durch die Gleichung $R=a^{-b}$ dargestellt werden kann, worin a und b Konstanten bedeuten.

4. Die Diffusionserscheinung der gesättigten Pikrinsäurelösung läuft parallel mit der Zeit. 


$$
\mathrm{R}=\mathrm{a}+\mathrm{bT}
$$

5. Die Diffusion der 3,44\%igen Kaliumbichromatlösung erfolgt regelmässig, d.h. mit der Zeit nimmt sie erst schnell, dann immer langsamer $\mathrm{zu}$.

$$
\mathrm{R}=\mathrm{C}+\frac{\mathrm{T}}{\mathrm{a}+\mathrm{bT}}
$$

6. Diese einfache Untersuchung zeigt, dass wir von einem fixierten Gewebestück eigentlich nie erwarten dürfen, dass es ganz dasselbe Bild wie im Leben zeigt.

Zum Schlusse möchte ich Herrn Professor T. Tomita für die Anregung zu dieser Arbeit und die wertvollen Ratschläge meinen besten Dauk aussprechen.

Ausserdem möchte ich Herrn Professor J. Ueberschaar und Professor T. Takenaka für ihre Revisionen meinen herzlichsten Dank ausdrücken.

(2. Oktober, 1929)

\section{Literatur.}

1. Bechhold, H. Die Kolloide in Biol. u. Med. 4. Aufl. 1922.

2. Bito, Mitteil. d. Med. Gesellschaf. zu Okayama. 443.

3. Fischer, A., Anat. Anz. Bd. 9. 1893-1895.

4. " " Bd. 10. 1894-1895.

5. " Fixierung, Färbung und Bau des Protoplasmas. 1899.

6. Fischer, H., Kolloidzeitschrift. 34. 1924.

7. Fischer, H., und Hooker Kolloidzeitschrift. 40. 1926.

8. Enzyklopädie d. mikrosk. Technick. 1910.

9. Hansmann, Zeitschrift f, allg. u. anorg. Chemie. 40. 1904.

10. J ableynski, K., Kolloidzeitschrift. 40. 1926.

11. Kohlrausch u. Holborn, Das Leitmögen d. Elektrolyte. 1916.

12. Lepeschkin, W., Kolloidchemie des Protoplasmas. 1924.

13. Meyer, Ar., Morph. u. physiol. Analyse d. Zelle der Pflanzen u. Tiere. 1920.

14. Ogura, K., Zukeisan oyobi Zuhyo. 1924.

15. Ostwald, W., Zeitschrift f. phy. Chemie. 3. 1888.

16. Scheffer, R., "

17. Schleussner, Kolloidzeitschrift. 31. 1922 .

18. Schäde, R., Protoplasma. 3. 1927.

19. Stoeltzner, H., Zeitschrift f. wiss. Mikrosk. 23. 1906.

20. Schaffer, J., Anat. Anz. 51. 181819.

21. Tomita u. Fujita, Nippon Kaibogaku Zasshi. 3. 1929.

22. Teruuchi, Mitteil. d. Med. Gessell. z. Osaka. 2. 1929.

23. Watanabe, M., Shizen Kagaku. 3. 1928.

24. Yamaha, G., Toyo Gakugei Zasshi. 45. 1929.

25.

44. 1928

26. $"$ The Botanical Magazine. April 1926.

27. Yasuzumi, Nippon Kaibogaku Zasshi. 2. 1929. 